\title{
Bound States for a Magnetic Impurity in a Superconductor
}

\author{
Zorawar S. Bassi ${ }^{\dagger}$ \\ André LeClair \\ Newman Laboratory \\ Cornell University \\ Ithaca, NY 14853, USA
}

\begin{abstract}
We discuss a solvable model describing an Anderson like impurity in a BCS superconductor. The model can be mapped onto an Ising field theory in a boundary magnetic field, with the Ising fermions being the quasi-particles of the Bogoliubov transformation in BCS theory. The reflection S-matrix exhibits Andreev scattering and the existence of bound states of the quasi-particles with the impurity lying inside the superconducting gap.
\end{abstract}

$\dagger$ zorawar@mail.lns.cornell.edu 


\section{Introduction}

The problem of magnetic impurities in superconductors is an old yet still very interesting problem. Starting with the work of Abrikosov and Gor'kov [1], numerous theoretical studies have addressed this problem. The models most frequently used to describe the magnetic impurities are the Kondo (s-d exchange) model [2] and some form of the Anderson model [3]. Most earlier works are based on a perturbative approach. A review of the earlier works and various references can be found in [4].

An important effect due to the impurities involves the existence of localized excited states within the superconducting energy gap. The results of Müller-Hartmann and Zittartz on gap states due to a Kondo impurity are well known [5]. More recent studies of the gap states include a numerical renormalization group analysis for a Kondo impurity [6], quantum Monte Carlo techniques applied to the symmetric Anderson model [7], large-N slave boson methods for an N-fold degenerate Anderson impurity [8] and a Bethe ansatz analysis for an Anderson impurity by Rupasov [9]. The closely related problem of an Anderson like impurity in a Hubbard chain, which has a spin gap, was studied in [10]. Recent experimental work on single impurities was reported in [11].

In this paper we consider a rather simplified version of the problem of a magnetic impurity in a BCS superconductor. The virtues of our model are that it is solvable by elementary methods, and also that it reveals some of the expected features of more complicated models. Our solution represents a self-consistent treatment of the BCS pair potential and a fully quantum impurity. We came to consider this model after a detailed study of the more difficult theory corresponding to a bulk mass term in the usual Kondo model [12]. Here, the interaction with the impurity is taken to be of the Anderson form. However unlike the Anderson model, the impurity ' $\mathrm{d}$ ' orbital is considered to have no spin (or orbital) degeneracy, hence it is described by a single fermion operator $d$ and its adjoint $d^{\dagger}$, with no spin index. Since the conduction electrons have spin, the impurity interaction for this model is intermediate to that of the Anderson model and the resonant level model [13] at the Toulouse point. We study the model by mapping it onto a boundary field theory (BFT) and solving for the reflection S-matrix (i.e. boundary scattering matrix). The BFT consists of two decoupled Ising models with boundary magnetic fields. The Ising fermions can be associated with the quasi-particles resulting from a Bogoliubov transformation. From this mapping one sees that whereas a Kondo impurity has no non-trivial flow in boundary conditions, an Anderson impurity does have a flow between free and fixed boundary conditions. A similar model, where the localized 'd' electrons do have a spin degeneracy, i.e. the Anderson model with no Coulomb repulsion $(U=0)$, was studied some time ago in [14] using the more conventional Green's functions approach.

Our solution exhibits a single-impurity version of Andreev scattering. Andreev scattering occurs at the boundary between a superconductor and an ordinary metal, wherein quasi-particles are reflected into quasi-holes. For a single magnetic impurity, superconductivity can be destroyed locally in the vicinity of the impurity by the magnetic field of the impurity, in analogy with the Meissner effect. Thus one is led to expect that the impurity reflects quasi-particles into quasi-holes, and our solution confirms this.

It is well-known that magnetic impurities compete with superconductivity in a manner analogous to the Meissner effect: if the concentration of impurities is large, enough bound states can open up in the gap that the gap disappears, destroying superconductivity. For a single impurity the competition is between the formation of Cooper pairs and the binding of electrons to the impurity, and is manifested in the properties of the bound states localized at the impurity. In our model, from the poles in the reflection amplitude for each Bogoliubov quasi-particle we can determine the bound states of the quasi-particle with the impurity. Simple energetics determines whether the bound state is stable. The impurity has a magnetic energy scale $E_{\text {imp. }}$. When the gap $\Delta$ is zero, this energy scale appears as a resonance in the reflection S-matrix, and corresponds to an unstable bound state of the original electrons with the impurity. (In the doubled boundary Ising description, this resonance corresponds to a splitting in the degeneracy of the two Ising ground states induced by the boundary magnetic field.) When the gap is non-zero, the state of energy $E_{\text {imp }}$ can become stable if it is forbidden to decay into the bulk superconductor because of the gap; this occurs if $\Delta>E_{\text {imp }}$. This bound state has an excitation energy, $E_{b}$, less than the superconducting gap $\Delta$. The bound state energy is a function of $E_{\mathrm{imp}}$ and $\Delta$, and is given in (3.15) below. We find that the bound states in the gap disappear when the strength of the impurity is large enough, i.e. when $E_{\mathrm{imp}} \geq \Delta$. In this case the 'Meissner effect' is reversed and superconductivity is favored: the quasi-particle-impurity bound state becomes unstable and it is more energetically favorable to form Cooper pairs. 


\section{The Model}

The model we use to describe an impurity in a superconductor consists of the (mean-field) BCS Hamiltonian and an Anderson like interaction,

$$
\begin{gathered}
H=H_{\mathrm{BCS}}+H_{I} \\
H_{\mathrm{BCS}}=\sum_{\vec{k} \sigma} \xi_{\vec{k}} c_{\vec{k} \sigma}^{\dagger} c_{\vec{k} \sigma}-\Delta \sum_{\vec{k}}\left(c_{\vec{k} \uparrow}^{\dagger} c_{-\vec{k} \downarrow}^{\dagger}+c_{-\vec{k} \downarrow} c_{\vec{k} \uparrow}\right) \\
H_{I}=\sum_{\vec{k} \sigma} V_{\vec{k}}\left(c_{\vec{k} \sigma}^{\dagger} d+d^{\dagger} c_{\vec{k} \sigma}\right)
\end{gathered}
$$

where $c_{\vec{k} \sigma}^{\dagger}$ are conduction electron creation operators of momentum $\vec{k}$ and spin $\sigma$ ( $\uparrow$ up and $\downarrow$ down), and $\Delta$ is the energy gap. The impurity is at the origin in three spacial dimensions. The operators $d$ and $d^{\dagger}$ describe a spinless fermion localized on the impurity, satisfying

$$
\left\{d, d^{\dagger}\right\}=1, \quad d^{2}=d^{\dagger}=0 .
$$

These operators act on a two-dimensional Hilbert space $|0\rangle_{d},|1\rangle_{d}$ where $|1\rangle_{d}=d^{\dagger}|0\rangle_{d}$. In the unperturbed theory these two states are taken to be degenerate in energy. Since the impurity level has no spin degeneracy, there is no Coulomb repulsion term as in the Anderson model. The energy $\xi_{\vec{k}}$ is measured relative to the fermi energy $\epsilon_{F}, \xi_{\vec{k}}=\epsilon_{\vec{k}}-\epsilon_{F}$, where $\epsilon_{\vec{k}}$ is the electron kinetic energy. In the impurity interaction $H_{I}, V_{\vec{k}}$ is the hybridization matrix element between the band states and the impurity states.

We can diagonalize the BCS part by a Bogoliubov unitary transformation [15]

$$
c_{\vec{k} \uparrow}=u_{\vec{k}} \tilde{b}_{\vec{k} 1}+\alpha_{\vec{k}} v_{\vec{k}} \tilde{b}_{\vec{k} 2}^{\dagger}, \quad c_{-\vec{k} \downarrow}^{\dagger}=u_{\vec{k}} \tilde{b}_{\vec{k} 2}^{\dagger}-\alpha_{\vec{k}} v_{\vec{k}} \tilde{b}_{\vec{k} 1},
$$

with

$$
\begin{gathered}
u_{\vec{k}}=\frac{1}{\sqrt{2}} \sqrt{1+\alpha_{\vec{k}} \frac{\xi_{\vec{k}}}{\omega_{\vec{k}}}}, \quad v_{\vec{k}}=\frac{1}{\sqrt{2}} \sqrt{1-\alpha_{\vec{k}} \frac{\xi_{\vec{k}}}{\omega_{\vec{k}}}} \\
\alpha_{\vec{k}}=\operatorname{sgn}\left(|\vec{k}|-k_{F}\right)= \begin{cases}1, & \text { if }|\vec{k}|>k_{F} ; \\
-1, & \text { if }|\vec{k}|<k_{F},\end{cases}
\end{gathered}
$$

and

$$
\omega_{\vec{k}}=\sqrt{\xi_{\vec{k}}^{2}+\Delta^{2}}
$$

In terms of the quasi-particle operators $\left(\tilde{b}_{\vec{k} j}^{\dagger}, \tilde{b}_{\vec{k} j}\right)$, which are often referred to as Bogoliubons, $H_{\mathrm{BCS}}$ becomes

$$
H_{\mathrm{BCS}}=\sum_{\vec{k} j} \tilde{\omega}_{\vec{k}} \tilde{b}_{\vec{k} j}^{\dagger} \tilde{b}_{\vec{k} j}
$$

where

$$
\tilde{\omega}_{\vec{k}}= \begin{cases}-\omega_{\vec{k}}, & \text { if }|\vec{k}|<k_{F} \\ \omega_{\vec{k}}, & \text { if }|\vec{k}|>k_{F} .\end{cases}
$$

The ground state of (1.9), $|0\rangle_{b}$, is obtained by filling all levels with $|\vec{k}|<k_{F}$. Rather than working with the $\tilde{b}$ 's, it is more convenient to introduce the following 'modified' quasi-particle operators

$$
b_{\vec{k} 1}=\left\{\begin{array}{ll}
\tilde{b}_{\vec{k} 1}, & \text { if }|\vec{k}|>k_{F} ; \\
\tilde{b}_{\vec{k} 2}, & \text { if }|\vec{k}|<k_{F},
\end{array} \quad b_{\vec{k} 2}= \begin{cases}\tilde{b}_{\vec{k} 2}, & \text { if }|\vec{k}|>k_{F} ; \\
\tilde{b}_{\vec{k} 1}, & \text { if }|\vec{k}|<k_{F} .\end{cases}\right.
$$

These operators satisfy canonical commutation relations and will simply be referred to as the quasi-particles of the system. The BCS Hamiltonian maintains its diagonal form (1.9) when expressed in this new basis. 
The Bogoliubov transformation applied to the interaction gives

$$
H_{I}=\frac{V}{\sqrt{2}} \sum_{\vec{k}}\left[\left(b_{\vec{k} 1}^{\dagger}-b_{\vec{k} 1}\right)\left(d+d^{\dagger}\right)+\left(b_{\vec{k} 2}^{\dagger}+b_{\vec{k} 2}\right)\left(d-d^{\dagger}\right)\right],
$$

where we have set $\vec{k}=\vec{k}_{F}$ in $V_{\vec{k}}, V=V_{k_{F}}$, and in the coefficients of the unitary transformation (1.6). In (1.12) we have kept the terms $b_{\vec{k} j}^{\dagger} d^{\dagger}$ and $b_{\vec{k} j} d$, which were omitted in the 'rotating wave approximation' made in [9].

Assuming the impurity to be point-like with only s-wave scattering, we expand the operators $\left(b_{\vec{k} j}^{\dagger}, b_{\vec{k} j}\right)$ in spherical harmonics and retain only the angular momentum $l=m=0$ terms (the $l \neq 0$ terms do not couple to the impurity). This gives the one dimensional Hamiltonian (sum over $j$ )

$$
H=\int_{-\infty}^{\infty} \frac{d k}{2 \pi}\left(\tilde{\omega}(k) b_{j}^{\dagger}(k) b_{j}(k)+\frac{V}{\sqrt{2}}\left[\left(b_{1}^{\dagger}(k)-b_{1}(k)\right)\left(d+d^{\dagger}\right)+\left(b_{2}^{\dagger}(k)+b_{2}(k)\right)\left(d-d^{\dagger}\right)\right]\right),
$$

where

$$
\tilde{\omega}(k)=\left\{\begin{array}{ll}
-\omega(k), & \text { if } k<0 ; \\
\omega(k), & \text { if } k>0,
\end{array} \quad \omega(k)=\sqrt{k^{2}+\Delta^{2}} .\right.
$$

We have linearized $\xi_{\vec{k}}$ about the fermi surface, $\xi_{\vec{k}}=|\vec{k}|-k_{F}$, setting $v_{F}=1$, and defined $k=|\vec{k}|-k_{F}$ to be the momentum relative to the fermi surface. The operators $b_{j}^{\dagger}(k)$ create quasi-particles of momentum $k+k_{F}$ and satisfy

$$
\left\{b_{i}(k), b_{j}^{\dagger}\left(k^{\prime}\right)\right\}=2 \pi \delta_{i j} \delta\left(k-k^{\prime}\right) .
$$

Note that $V$ has dimension $\sqrt{\text { energy; }}$ below we show that the scale $E_{\text {imp }}$ introduced in the introduction is simply $V^{2}$.

A final transformation $i b_{1}(k) \rightarrow b_{1}(k)$ and $-i b_{1}^{\dagger}(k) \rightarrow b_{1}^{\dagger}(k)$, allows $H$ to be rewritten as

$$
\begin{gathered}
H=H_{1}+H_{2} \\
H_{j}=\int_{-\infty}^{\infty} \frac{d k}{2 \pi}\left(\tilde{\omega}(k) b_{j}^{\dagger}(k) b_{j}(k)+i \frac{V}{\sqrt{2}}\left(b_{j}^{\dagger}(k)+b_{j}(k)\right) a_{j}\right) \quad(j=1,2),
\end{gathered}
$$

where

$$
a_{1}=\left(d+d^{\dagger}\right), \quad a_{2}=-i\left(d-d^{\dagger}\right) .
$$

The operators $a_{j}$ anticommute with the $b$ operators and satisfy

$$
a_{j}^{\dagger}=a_{j}, \quad a_{j}^{2}=1, \quad\left\{a_{1}, a_{2}\right\}=0,
$$

implying $\left[H_{1}, H_{2}\right]=0$. Thus we only need to concentrate on one copy and henceforth drop the subscript.

\section{Mapping onto a Boundary Field Theory}

In this section we map (1.17) onto a boundary Ising field theory. As in the usual Kondo model, s-wave scattering has reduced the problem to a one-dimensional problem on the half-line $r \geq 0$, where $r$ is the spherical radial coordinate. Henceforth, $r$ is designated as the spacial variable $-x$.

The action for an Ising field theory on the half-line with a magnetic field at the boundary is [16]

$$
\begin{gathered}
S=S_{\mathrm{bulk}}+S_{\mathrm{a}-\text { free }}+S_{\mathrm{bc}}+S_{\mathrm{int}} \\
S_{\mathrm{bulk}}=\frac{i}{2} \int_{-\infty}^{\infty} d t \int_{-\infty}^{0} d x\left(\psi\left(\partial_{t}-\partial_{x}\right) \psi+\bar{\psi}\left(\partial_{t}+\partial_{x}\right) \bar{\psi}+2 \Delta \psi \bar{\psi}\right)
\end{gathered}
$$




$$
\begin{gathered}
S_{\mathrm{bc}}=-\left.\frac{i}{2} \int_{-\infty}^{\infty} d t \psi \bar{\psi}\right|_{x=0}, \quad S_{\mathrm{a}-\text { free }}=\frac{i}{2} \int_{-\infty}^{\infty} d t a(t) \partial_{t} a(t) \\
S_{\mathrm{int}}=-\left.i \frac{V}{\sqrt{2}} \int_{-\infty}^{\infty} d t(\psi+\bar{\psi}) a(t)\right|_{x=0} .
\end{gathered}
$$

The term $S_{\text {bulk }}$ is simply a free Majorana action with $\Delta$ being the fermion mass, $S_{\mathrm{bc}}$ serves to enforce the free boundary condition

$$
\psi=\bar{\psi} \quad \text { at } x=0,
$$

for $V=0$, and $S_{\text {int }}$ gives the interaction between the boundary spins and the boundary magnetic field $V$. The fermionic field $a(t)$, which anticommutes with $(\psi, \bar{\psi})$, describes the ground state degeneracy due to the two different expectation values of the spin field and satisfies $a^{2}=1$. Thus $S_{\text {a-free }}$ is the kinetic term for $a(t)$. The bulk equations of motion are

$$
\left(\partial_{t}-\partial_{x}\right) \psi+\Delta \bar{\psi}=0, \quad\left(\partial_{t}+\partial_{x}\right) \bar{\psi}-\Delta \psi=0 .
$$

Mode expansions satisfying (2.6) can be written as

$$
\left(\frac{\psi}{\psi}\right)=\int_{-\infty}^{\infty} \frac{d k}{2 \pi}\left[\left(\begin{array}{c}
u(k) \\
v(k)
\end{array}\right) A(k) e^{-i \vec{k} \cdot \vec{x}}+\left(\begin{array}{c}
u^{*}(k) \\
v^{*}(k)
\end{array}\right) A^{\dagger}(k) e^{i \vec{k} \cdot \vec{x}}\right]
$$

where

$$
\begin{gathered}
\left\{A(k), A^{\dagger}\left(k^{\prime}\right)\right\}=2 \pi \delta\left(k-k^{\prime}\right) \\
u(k)=e^{-i \pi / 4} \sqrt{\frac{\omega(k)-k}{2 \omega(k)}}, \quad v(k)=e^{i \pi / 4} \sqrt{\frac{\omega(k)+k}{2 \omega(k)}},
\end{gathered}
$$

and $\vec{k} \cdot \vec{x}=\omega(k) t-k x, \omega(k)=\sqrt{k^{2}+\Delta^{2}}$.

The Ising Hamiltonian associated with (2.1) takes the form

$$
H=\int_{-\infty}^{\infty} \frac{d k}{2 \pi}\left(\omega(k) A^{\dagger}(k) A(k)+i \frac{V}{\sqrt{2}}\left(g(k) A(k)+g^{*}(k) A^{\dagger}(k)\right) a\right),
$$

where

$$
g(k)=u(k)+v(k) .
$$

The second term in (2.10) gives the boundary interaction. Comparing (2.10) with (1.17), we can map each $H_{j}$ onto the Ising Hamiltonian if we relate the $b$ operators to the $A$ operators as follows

$$
\begin{gathered}
b(k)=g(k) A(k) \quad \text { if } k>0 \\
b(k)=g^{*}(k) A^{\dagger}(k) \quad \text { if } k<0,
\end{gathered}
$$

and identify $a_{j}$ with $a$. The quasi-particles correspond to the Ising fermions and the impurity coupling $V$ plays the role of a boundary magnetic field. Using

$$
g(k) g^{*}(k)=1,
$$

it is easily shown that the $b$ operators satisfy canonical commutation relations

$$
\left\{b(k), b^{\dagger}\left(k^{\prime}\right)\right\}=2 \pi \delta\left(k-k^{\prime}\right) .
$$

\section{Quasi-Particle Bound States}

Having mapped (1.17) onto the BFT (2.1), the impurity interaction can then be described by the reflection S-matrix (boundary scattering matrix) for the fermion operators. We compute the reflection 
amplitude from the action as discussed in [16]. The boundary terms in the variation of (2.1) lead to the boundary equation of motion (at $x=0$ )

$$
-\partial_{t}(\psi-\bar{\psi})=V^{2}(\psi+\bar{\psi}) .
$$

Here we see the flow between free $(V=0)$ and fixed $(V=\infty)$ boundary conditions. Interpreting (3.1) to hold when acting on a formal boundary operator $B$, we substitute the mode expansions and define the reflection S-matrix, or reflection amplitude, $R(k)$, through

$$
A^{\dagger}(k) B=R(k) A^{\dagger}(-k) B .
$$

The reflection amplitude for the Ising model was computed in [16]. Let us parametrize the energy and momentum with the rapidity $\theta$

$$
\omega(\theta)=\Delta \cosh \theta, \quad k(\theta)=\Delta \sinh \theta .
$$

Define $A(\theta)=A(k) \sqrt{\omega(k)}$, satisfying $\left\{A(\theta), A^{\dagger}\left(\theta^{\prime}\right)\right\}=2 \pi \delta\left(\theta-\theta^{\prime}\right)$. We find

$$
A^{\dagger}(\theta) B=R(\theta) A^{\dagger}(-\theta) B
$$

where

$$
R(\theta)=-i \tanh \left(i \frac{\pi}{4}-\frac{\theta}{2}\right) R_{b}(\theta), \quad R_{b}(\theta)=\left(\frac{i \sinh \theta+\left(V^{2} / \Delta-1\right)}{i \sinh \theta-\left(V^{2} / \Delta-1\right)}\right) .
$$

The amplitude $R_{b}$ is the reflection amplitude for the Bogoliubov operators $b(k)$

$$
b^{\dagger}(k>0) B=R_{b}(\theta) b(-k) B, \quad b(k<0) B=R_{b}(\theta) b^{\dagger}(-k) B .
$$

Defining the quasi-electron operators $e(k)$ for $k>0$ and quasi-hole operators $h(k)$ for $k<0$

$$
e(k)=b(k), \text { for } k>0 ; \quad h(k)=b^{\dagger}(k), \text { for } k<0,
$$

then (3.6) can be expressed as

$$
e^{\dagger}(k) B=R_{b}(k) h^{\dagger}(-k) B, \quad h^{\dagger}(k) B=R_{b}(k) e^{\dagger}(-k) B .
$$

The quasi-electrons scatter into quasi-holes and vice-versa at the boundary. This is a form of Andreev reflection, which usually occurs at the boundary between a superconductor and a normal metal. Here the boundary is a single impenetrable impurity, i.e. there is no transmission. For the original quasi-particles, the $\tilde{b}$ 's, equation (3.8) implies that quasi-electrons of one type, say $\tilde{b}_{1}^{\dagger}(k)$, scatter into quasi-holes of the other type $\tilde{b}_{2}(-k)$.

In terms of the energy $E(\theta)=\omega(\theta), R_{b}$ can be written as

$$
R_{b}(E)=-\frac{E^{2}+\left(V^{2}\right)^{2}-2 \Delta V^{2}}{\left(V^{2}\right)^{2}-E^{2}-2\left(V^{2}-\Delta\right)\left(\Delta+i \sqrt{E^{2}-\Delta^{2}}\right)} .
$$

For $\Delta=0,(3.9)$ becomes

$$
R_{b}^{0}(E)=-\frac{E_{\mathrm{imp}}^{2}+E^{2}}{E_{\mathrm{imp}}^{2}-E^{2}-i \Gamma E}
$$

where

$$
E_{\text {imp }}=V^{2}, \quad \Gamma=2 V^{2} .
$$

The energy $E_{\mathrm{imp}}$ is a 'magnetic energy' scale associated with the magnetic impurity. This becomes clearer in the Ising picture where $V$ effectively acts as a local magnetic field. Near $E \approx E_{\text {imp }}, R_{b}^{0}(E)$ takes a Lorentzian form

$$
R_{b}^{0}(E)=-i \frac{(\Gamma / 2)^{2}}{\left(E_{\mathrm{imp}}-E\right)^{2}+(\Gamma / 2)^{2}} .
$$


From (3.12) we see that when $\Delta=0$, there is a resonance in the reflection amplitude at $E=E_{\text {imp }}$ with a width $\Gamma$. This resonance corresponds to an unstable electron-impurity state with an energy $E_{\text {imp }}$ and decay rate $\Gamma$. If the gap $\Delta$ is non-zero, then this state can become a stable bound state (see below).

As with the bulk S-matrix, poles of the reflection S-matrix provide information on bound states. Poles in the physical strip $0 \leq \operatorname{Im} \theta \leq \pi$ can be indicative of either bulk bound states or boundary bound states. Since the bulk theory is a free fermion theory with trivial scattering $(S=-1)$, we only have to consider the latter case. The quasi-particle reflection amplitude is

$$
R_{b}(\theta)=\frac{i \sinh \theta+\left(E_{\mathrm{imp}} / \Delta-1\right)}{i \sinh \theta-\left(E_{\mathrm{imp}} / \Delta-1\right)}
$$

We will now express all quantities in terms of the physical energy scale $E_{\mathrm{imp}}$. Writing $\theta$ as $i u$, poles of $R_{b}(\theta)$ occur when

$$
\sin u=1-\frac{E_{\mathrm{imp}}}{\Delta} .
$$

Restricting $u$ to be in the physical strip, there is a single pole at $u=u_{b}$ satisfying (3.14) provided $E_{\text {imp }} \leq \Delta$. (Note that there is also a pole at $\tilde{u}_{b}=\pi-u_{b}$, corresponding to scattering in the cross channel. The results that follow also apply to this pole.) Thus a quasi-particle forms a bound state with the impurity if $E_{\mathrm{imp}} \leq \Delta$. This is the same state that appears as a resonance in (3.12) for a vanishing gap; it has become stable because its decay into the bulk is forbidden if $E_{\mathrm{imp}}<\Delta$. The excitation energy (above the ground state) of the bound state, $E_{b}$, is

$$
E_{b}=\Delta \cos u_{b}=\sqrt{\Delta E_{\mathrm{imp}}\left(2-\frac{E_{\mathrm{imp}}}{\Delta}\right)} .
$$

For $E_{\text {imp }}<\Delta$, the excitation energy lies within the superconducting gap $\left(E_{b}<\Delta\right)$ and the bound state is stable. When $E_{\mathrm{imp}}>\Delta$, the excitation energy falls in the continuum and there is no bound state.

If $E_{\mathrm{imp}}=0$, the pole occurs at $i \pi / 2$, implying that the ground state is degenerate. This makes sense since for zero interaction the ground states are

$$
|0\rangle_{b} \otimes|0\rangle_{d} \text { and }|0\rangle_{b} \otimes|1\rangle_{d}
$$

where $|0\rangle_{b}$ is the quasi-particle ground state. In terms of the quasi-electron and quasi-hole operators $(e(k), h(-k))$ with $k>0,|0\rangle_{b}$ is a zero energy state satisfying

$$
e(k)|0\rangle_{b}=h(-k)|0\rangle_{b}=0 .
$$

As $E_{\text {imp }}$ increases above zero, the degeneracy is lifted by the interaction. The states (3.16) are split into a ground state and the bound state with energy (3.15). In the Ising picture the degenerate ground states, $|0, \pm\rangle_{I}$, are labeled by the expectation values of the spin field $\sigma(x),\langle\sigma(x)\rangle_{ \pm}= \pm \bar{\sigma}$, where $\bar{\sigma}$ is the spontaneous magnetization. A non-vanishing magnetic field, $\sqrt{E_{\mathrm{imp}}}>0$, removes the degeneracy of the free boundary spin states $|0, \pm\rangle_{I}$.

Based on the above discussion, we can interpret Andreev reflection of the quasi-particles as follows. Suppose we extend our system by adjoining a lattice of impurities for $x>0$. In the region $x>0$ superconductivity is more or less destroyed and we have a superconductor-normal conductor boundary at $x=0$. In this case there will be Andreev reflection at the boundary, as well as transmission. A lattice consisting of a single impurity then behaves as a superconductor-normal conductor barrier with the probability for Andreev reflection unity, $\left|R_{b}\right|^{2}=1$, just as would be the case for subgap particles incident on a superconductor.

So far we have only been dealing with one of the Hamiltonians $H_{j}$ in (1.16). Each type of quasi-particle, $b_{1}^{\dagger}(k)$ or $b_{2}^{\dagger}(k)$, will form a bound state. If we represent the ground state of $H_{j}$ by $|0\rangle_{j}$ (for small $E_{\text {imp }}>0$ ) and the bound state by $|b\rangle_{j}$, then the ground state of $H=H_{1}+H_{2},|0\rangle$, will be

$$
|0\rangle=|0\rangle_{1} \otimes|0\rangle_{2},
$$

and the excited states are

$$
|1\rangle=|b\rangle_{1} \otimes|0\rangle_{2}, \quad\left|1^{\prime}\right\rangle=|0\rangle_{1} \otimes|b\rangle_{2}
$$


both with the same energy and lying inside the gap.

\section{Conclusions}

We have shown that the model (1.1) is a solvable system, which is equivalent to two decoupled Ising models with boundary magnetic fields. From the boundary field theory we calculated the reflection amplitudes for the quasi-particles. At the boundary the quasi-particles are Andreev reflected. Provided the magnetic energy $E_{\text {imp }}$ (or equivalently the hybridization matrix element/magnetic field $V$ ) is not too large, bound states exist, consisting of a quasi-particle bound to the impurity. The energy of the stable bound states is less than the gap, $E_{b}<\Delta$.

We hope this calculation further motivates the use of techniques common to integrable systems for the study of impurities in superconductors. It would be interesting to apply the techniques discussed here, and in [12], to more complicated systems, such as the original Anderson model, or magnetic impurities in $d$-wave superconductors studied in [17].

\section{Acknowledgments}

We would like to thank V. Ambegaokar, A. Clerk and E. Fradkin for discussions. This work is supported in part by the National Science Foundation and the National Young Investigator program. Z. S. Bassi also acknowledges support from the Olin Foundation.

\section{References}

[1] A. A. Abrikosov and L. P. Gor'kov, Sov. Phys. JETP 12 (1961) 1243 [Zh. Eksp. Teor. Fiz. 39 (1960) 1781].

[2] J. Kondo, Prog. Theor. Phys. 32 (1964) 37.

[3] P. W. Anderson, Phys. Rev. 124 (1961) 41.

[4] S. V. Vonsovsky, Yu A. Izyumov and E. Z. Kurmaev, Superconductivity of Transition Metals, SpringerVerlag, 1982.

[5] E. Müller-Hartmann and J. Zittartz, Z. Phys. 232 (1970) 11; ibid. 234 (1970) 58; J. Zittartz, Z. Phys. 237 (1970) 419; E. Müller-Hartmann, "Recent Theoretical Work on Magnetic Impurities in Superconductors" in Magnetism, Vol. 5, Ed. H. Suhl, Academic Press, 1973.

[6] K. Satori, H. Shiba, O. Sakai and Y. Shimizu, J. Phys. Soc. Jpn. 61 (1992) 3239; ibid. 62 (1993) 3181.

[7] M. Jarrell, D. S. Sivia and B. Patton, Phys. Rev. B 42 (1990) 4804; W. Chung and M. Jarrell, Phys. Rev. Lett. 77 (1996) 3621.

[8] L. S. Borkowski and P. J. Hirschfeld, J. Low Temp. Phys. 96 (1994) 185.

[9] V. I. Rupasov, Phys. Lett. A 237 (1997) 80.

[10] A. A. Zvyagin and P. Schlottmann, Phys. Rev. B 56 (1997) 300.

[11] A. Yazdani, B. A. Jones, C. P. Lutz, M. F. Crommie and D. M. Eigler, Science 275 (1997) 1767.

[12] Z. S. Bassi and A. LeClair, Nucl. Phys. B 552 (1999) 643.

[13] V. M. Filyov and P. B. Wiegmann, Phys. Lett. A 76 (1980) 283.

[14] K. Machida and F. Shibata, Prog. Theor. Phys. 47 (1972) 1817.

[15] M. Tinkham, Introduction to Superconductivity, McGraw-Hill, Inc., 1996.

[16] S. Ghoshal and A. Zamolodchikov, Int. J. Mod. Phys. A 9 (1994) 3841.

[17] C. R. Cassanello and E. Fradkin, Phys. Rev. B 56 (1997) 11246. 